8-1992

\title{
Closed-Orbit Theory of Oscillations in Atomic Photoabsorption Cross Sections in a Strong Electric Field. II. Derivation of Formulas
}

J. Gao

William \& Mary

John B. Delos

William \& Mary, jbdelos@wm.edu

Follow this and additional works at: https://scholarworks.wm.edu/aspubs

Part of the Physics Commons

\section{Recommended Citation}

Gao, J. and Delos, John B., Closed-Orbit Theory of Oscillations in Atomic Photoabsorption Cross Sections in a Strong Electric Field. II. Derivation of Formulas (1992). Physical Review A, 46(3), 1455-1467.

https://doi.org/10.1103/PhysRevA.46.1455

This Article is brought to you for free and open access by the Arts and Sciences at W\&M ScholarWorks. It has been accepted for inclusion in Arts \& Sciences Articles by an authorized administrator of W\&M ScholarWorks. For more information, please contact scholarworks@wm.edu. 


\title{
Closed-orbit theory of oscillations in atomic photoabsorption cross sections in a strong electric field. II. Derivation of formulas
}

\author{
J. Gao and J. B. Delos \\ Physics Department, College of William and Mary, Williamsburg, Virginia 23187
}

(Received 3 December 1991)

\begin{abstract}
A formula for photoabsorption cross sections of hydrogen and alkali-metal atoms in a static electric field is derived, based on the closed-orbit theory previously used to study hydrogen in a magnetic field. Electric fields are simpler than magnetic fields, because the classical motion is regular and closed orbits can be enumerated. In alkali metals the core modifies the relevant dipole matrix elements, and it produces additional phase shifts.
\end{abstract}

PACS number(s): 32.60. $+\mathrm{i}, 32.80 . \mathrm{Cy}, 32.80 . \mathrm{Fb}$

\section{INTRODUCTION}

This is the second of our papers dealing with the effect of closed classical orbits on the absorption spectrum of atoms in a strong electric field. In the first paper [1] we stated a formula that connects closed orbits with oscillations in the absorption spectrum of hydrogen and sodium. We made comparison between theory and experiment above threshold. Here we present a complete theoretical analysis. The derivation is based on the theory developed in Ref. [2]. The approximations and physical picture used there are readily applied here.

The Schrödinger equation for a hydrogen atom in an electric field is separable in parabolic coordinates. Hence, unlike the case of an atom in a magnetic field, the classical trajectories are not chaotic. In principle, we can list all of the closed orbits that propagate away from and return to the vicinity of the nucleus. For hydrogen three approximations are used: (1) Near the atomic nucleus, the electric field is negligible; (2) far from the nucleus, the waves propagate semiclassically; and (3) returning waves are similar to (cylindrically modified) Coulomb-scattering waves.

For alkali-metal atoms, the potential energy felt by the electron is Coulombic outside a core, and hence the radial wave functions are hydrogenic functions with a phase shift $\delta_{l}$. In addition, $l-s$ coupling in the $3 p$ configuration mixes $l_{z}$ and $s_{z}$ into $\left(j, m_{j}\right)$ states. These two effects modify the angular distribution of the outgoing waves. Moreover, when the wave returns to the ion core, it is scattered. The scattered waves consist of a Coulombscattered wave and a core-scattered wave as well. Both of these waves then retrace the closed orbit and later return to the ion to produce oscillations which are associated with repetitions of the orbit. In other respects, the cross section is similar to that obtained for hydrogen.

The paper is organized as follows. In Sec. II we briefly explain the theory for atoms in magnetic fields given in Ref. [2]. In Secs. III-V the behavior of waves in the vicinity of the nucleus is discussed. We specify the initial wave function and the dipole operator, then the wave function near the ionization threshold and the radial integral, and finally the outgoing waves and the smooth background. In Sec. VI we discuss the semiclassical waves in the region where electric field and Coulomb field are comparable. The amplitude and the phase of wave functions are specified, and the effects of repetitions of classical closed orbits are taken into account. In Sec. VII the result of Secs. III-VI are put together to describe oscillations in the spectrum. In Sec. VIII we note that the formula cannot be applied to the $\theta=0$ orbit, and we derive an expression for this case. In Sec. IX, by using scaled variables to describe the properties of classical trajectories, we are able to get a simple analytic expression for the oscillator-strength density [3].

The accompanying paper examines the application of this theory in the simplest case: $E>0$, for which only one closed orbit exists. In future papers we will use the theory to calculate cross sections for $E<0$ [4].

\section{GENERAL FORMULA AND MODIFICATIONS}

In Ref. [2] the formula for the average oscillatorstrength density $D f(E)$ in a magnetic field was given. We follow closely the formulation given in Ref. [2], denoted here as "II," and equations from that paper are referenced as, for example, [II, (5.13a)]. We restrict ourselves here to the case where the light is linearly polarized along the electric field ( $\pi$-polarized light).

The photoabsorption cross section $\sigma(E)$ is related to the average oscillator-strength density $D f(E)$ by

$$
\sigma=\frac{2 \pi^{2} e^{2}}{m_{e} c} D f(E),
$$

and the oscillator-strength density is related to the Green's function by

$$
D f(E)=-\frac{2 m_{e}\left(E-E_{i}\right)}{\pi \hbar}\left\langle D \psi_{i}\left|\operatorname{Im} \hat{G}^{+}\right| D \psi_{i}\right\rangle .
$$

By dividing the Green's function into a "direct" part and a "returning" part and by using a semiclassical approximation, it was shown in Ref. [2] that the oscillatorstrength density could be written as a smooth"background" term plus a sum of sinusoidal oscillations, 


$$
D f(E)=D f_{0}(E)+\sum_{k} C_{k}(E) \sin \Delta_{k}(E) .
$$

The smooth-background term $D f_{0}(E)$ is equal to the oscillator-strength density that would be obtained in the absence of a magnetic field,

$D f_{0}(E=0)=-\frac{4 m_{e}\left(E-E_{i}\right)}{\hbar^{2}} \sum_{l^{\prime}}\left|b_{l^{\prime} m}^{i} I\left(n, l, l^{\prime}\right)\right|^{2}$.

The integrals $I\left(n, l, l^{\prime}\right)$ are dipole matrix elements between the initial bound state and the free states, and the $b_{l^{\prime} m}^{i}$ are Clebsch-Gordan coefficients. Each oscillatory term arises from a closed orbit labeled $k$. The amplitude and phase constant $C_{k}$ and $\Delta_{k}$ are calculated from the formula [II, (5.13a)] in atomic units

$$
\begin{aligned}
C_{k} \exp \left(i \Delta_{k}\right)= & \left(E-E_{i}\right) 2^{19 / 4} \pi^{3 / 2} r_{b}^{-1 / 4} \\
& \times\left(\sin \theta_{i}^{k} \sin \theta_{f}^{k}\right)^{1 / 2} A_{2}^{k} e^{-i(3 / 4) \pi} \\
& \times e^{2 i\left(8 r_{b}\right)^{1 / 2}} \exp \left[i\left(S_{2}^{k} / \hbar-\frac{1}{2} \pi \mu^{k}\right)\right] \\
& \times y\left(\theta_{i}^{k}\right) y^{*}\left(\theta_{f}^{k}\right),
\end{aligned}
$$

where

$$
\mathscr{y}(\theta)=\sum_{l_{1}}(-1)^{l_{1}} I\left(n, l, l_{1}\right) b_{l_{1} m}^{i} Y_{l_{1} m}(\theta, 0)
$$

and

$$
I\left(n, l, l_{1}\right)=\int_{0}^{\infty} R_{n l}(r) r^{3} \frac{J_{2 l_{1}+1}\left((8 r)^{1 / 2}\right)}{r^{1 / 2}} d r .
$$

Here $\theta_{i}^{k}$ and $\theta_{f}^{k}$ are the initial and final angles of the $k$ th closed orbit, $S_{2}^{k}+2\left(8 r_{b}\right)^{1 / 2}$ is the classical action around the orbit, $\mu^{k}$ is the Maslov index, $A_{2}^{k}$ is the classical amplitude, and $r_{b}$ is an arbitrary boundary radius which we typically take to be around $50 a_{0} . C_{k}$ and $\Delta_{k}$ are obtained by evaluating the amplitude and the phase of the righthand side of Eq. (2.5). For further details, see Ref. [2].

The above formula was derived to describe the absorption spectrum of hydrogen in a magnetic field. To describe the spectrum of hydrogen and alkali-metal atoms in electric fields, several modifications of the formula must be made. First, we need to modify the initial wave function; for hydrogen in electric fields the initial state is a combination of $s$ and $p$ states, while for sodium, the effect of spin-orbit coupling must be incorporated. Second, for alkali-metal atoms, each of the continuum wave functions $\psi_{n l m}$ is a hydrogenic function with a radial phase shift $\delta_{l}=\pi \mu_{l}$ caused by an atomic core. Third, we need to include repetitions of the closed orbits. Fourth, the formula does not apply to the orbit that goes up and down the field with $\theta_{i}=\theta_{f}=0$.

\section{WAVE FUNCTIONS OF THE INITIAL STATE}

Ionization cross sections depend upon the initial state from which the atom is ionized.

\section{A. Hydrogen}

In the experiment on hydrogen reported in Ref. [6], the electron was first excited to the $n=2$ shell and then ionized. Both steps were performed with $z$-polarized light. The external field couples the degenerate $s$ and $p$ states in the $n=2$ shell (linear Stark effect), so the initial wave function is

$$
\psi_{i}=\sum_{l} d_{l} R_{n l}(r) Y_{l m}(\theta, \varphi),
$$

or, more specifically,

$$
\psi_{i}=\frac{1}{\sqrt{2}}\left[R_{20} Y_{00}(\theta, 0)+\lambda R_{21} Y_{10}(\theta, 0)\right],
$$

in which $\lambda=1$ for the $2 s+2 p$ state and $\lambda=-1$ for the $2 s-2 p$ state; thus,

$$
d_{0}=\frac{1}{\sqrt{2}}, \quad d_{1}=\lambda \frac{1}{\sqrt{2}}
$$

Combining the initial state with the $z$ component of the dipole operator, one obtains for $\left|D \psi_{i}\right\rangle$ a combination of partial waves times Clebsch-Gordan coefficients. The result is written in the form

$$
D \psi_{i}=\sum_{l} d_{l} R_{n l} r\left[\sum_{l^{\prime}} b_{l^{\prime} m}^{l} Y_{l^{\prime} m}(\theta, \varphi)\right] \text {. }
$$

The coefficients are defined as

$$
b_{l^{\prime} m}^{l}=\int Y_{l^{\prime} m}^{*}(\theta, \varphi) \cos \theta Y_{l m}(\theta, \varphi) \sin \theta d \theta d \varphi,
$$

and the values needed in this paper are

$b_{00}^{1}=\sqrt{\frac{1}{3}}, \quad b_{10}^{0}=\sqrt{\frac{1}{3}}, \quad b_{20}^{1}=\sqrt{\frac{4}{15}}, \quad b_{21}^{1}=\sqrt{\frac{1}{15}}$.

For hydrogen, then,

$D \psi_{i}=(1 / \sqrt{2})\left[r R_{20} \sqrt{\frac{1}{3}} Y_{10}+\lambda r R_{21}\left(\sqrt{\frac{1}{3}} Y_{00}+\sqrt{\frac{4}{15}} Y_{20}\right)\right]$.

\section{B. Pseudohydrogen}

In his studies of the Stark effect, Harmin [5] used an artificial model of hydrogen, in which the coupling between $s$ and $p$ states was ignored. (His purpose was to compare hydrogen with alkali-metal atoms, which do not have this degeneracy.) To compare our results with his, we also consider this model, which we call "pseudohydrogen." It is just like real hydrogen, except that the initial states are

$$
\psi_{i}=R_{n l}(r) Y_{l m}(\theta, \varphi)
$$

and

$$
D \psi_{i}=r R_{n l}(r) \sum_{l^{\prime}} b_{l^{\prime} m}^{l^{\prime}} Y_{l^{\prime} m}(\theta, \varphi) .
$$

For the initial $3 p$ state, we have

$$
\psi_{i}=R_{31}(r) Y_{10}(\theta, \varphi)
$$

and

$$
D \psi_{i}=r R_{31}(r)\left(\sqrt{\frac{4}{15}} Y_{20}+\sqrt{\frac{1}{3}} Y_{00}\right) .
$$




\section{Sodium}

In the experiments on sodium [6], again the electron was first excited from $3 s$ to $3 p$ states using a laser that was linearly polarized parallel to the electric field. Since the spin-orbit splitting $\left(17 \mathrm{~cm}^{-1}\right)$ exceeded the laser resolution $\left(0.3 \mathrm{~cm}^{-1}\right)$, the laser selected either the ${ }^{3} P_{3 / 2}$ or the ${ }^{3} P_{1 / 2}$ state with the ionizing energy [7] $E_{3 / 2}=-24476.3 \mathrm{~cm}^{-1}$ and $E_{1 / 2}=-24493.5 \mathrm{~cm}^{-1}$. The initial-state wave function is then either

$R_{31}(r)\left(\sqrt{\frac{2}{3}} Y_{10} \alpha+\sqrt{\frac{1}{3}} Y_{11} \beta\right)$ for $j=\frac{3}{2}, m_{j}=\frac{1}{2}$

or

$R_{31}(r)\left(\sqrt{\frac{1}{3}} Y_{10} \alpha-\sqrt{\frac{2}{3}} Y_{11} \beta\right)$ for $j=\frac{1}{2}, m_{j}=\frac{1}{2}$

or the analogous states having $m_{j}=-\frac{1}{2}$. $\alpha$ and $\beta$ are two spin states, with

$$
\alpha=\left(\begin{array}{l}
1 \\
0
\end{array}\right), \quad \beta=\left(\begin{array}{l}
0 \\
1
\end{array}\right) \text {. }
$$

Generalizing this case, the initial situation corresponds to an incoherent statistical mixture of two pure states: Both have the same value of $j$, one has $m_{j}=\frac{1}{2}$, and the other has $m_{j}=-\frac{1}{2}$. Each of these can be written as

$$
\psi_{i}=\sum_{m} C_{m}^{j} R_{n l}(r) Y_{l m}(\theta, \varphi) \chi_{m_{j}-m}
$$

where $\chi_{1 / 2}=\alpha, \chi_{-1 / 2}=\beta$, and

$$
\begin{array}{ll}
C_{0}^{3 / 2}=\sqrt{\frac{2}{3}}, & C_{1}^{3 / 2}=\sqrt{\frac{1}{3}}, \\
C_{0}^{1 / 2}=\sqrt{\frac{1}{3}}, & C_{1}^{1 / 2}=-\sqrt{\frac{2}{3}} .
\end{array}
$$

Hence, for sodium,

$$
\begin{gathered}
\quad D \psi_{i}=\sum_{m} C_{m}^{j} r R_{n l}\left(\sum_{l^{\prime}} b_{l^{\prime} m}^{l} Y_{l^{\prime} m}(\theta, \varphi) \chi_{m_{j}-m}\right) ; \\
\text { if } j=\frac{3}{2}, m_{j}=\frac{1}{2} \\
D \psi_{i}=r R_{31}\left(\sqrt{\frac{8}{45}} Y_{20} \alpha+\sqrt{\frac{2}{9}} Y_{00} \alpha+\sqrt{\frac{1}{15}} Y_{21} \beta\right)
\end{gathered}
$$

or if $j=\frac{1}{2}, m_{j}=\frac{1}{2}$,

$$
D \psi_{i}=r R_{31}\left(\sqrt{\frac{4}{45}} Y_{20} \alpha+\sqrt{\frac{1}{9}} Y_{00} \alpha-\sqrt{\frac{2}{15}} Y_{21} \beta\right)
$$

\section{WAVE FUNCTION NEAR THE IONIZATION THRESHOLD AND RADIAL INTEGRAL}

For hydrogen (and pseudohydrogen) the wave functions near the ionization threshold were given in Eqs. [II, (4.4)-(4.11)], and the relevant dipole matrix elements are also defined in Eq. (2.7) of this paper. The values needed are

$$
\begin{aligned}
& I(2,0,1)=4.6888, \\
& I(2,1,0)=1.3535, \\
& I(2,1,2)=5.4142, \\
& I(3,1,2)=11.1282, \\
& I(3,1,0)=3.7094 .
\end{aligned}
$$

[The value of $I(3,1,0)$ given in Ref. [2] was not correct.]

To describe the wave functions for $\mathrm{Na}$, we must divide space into several regions: (1) For $r_{0} \lesssim 1 a_{0}$, inside the core, the wave function cannot be expressed in any simple form; it must be computed by some $a b$ initio method. However, we do not need much information about this region. (2) For $1 a_{0} \lesssim r \lesssim 50 a_{0}$, the wave functions are written in terms of the regular and irregular Coulomb function for the excited electron,

$R_{l}^{0, \mathrm{reg}}(r)=\cos \delta_{l} \frac{J_{2 l+1}(\sqrt{8 r})}{\sqrt{8 r}}-\sin \delta_{l} \frac{Y_{2 l+1}(\sqrt{8 r})}{\sqrt{8 r}}$,

$R_{l}^{0, \text { irreg }}(r)=\cos \delta_{l} \frac{Y_{2 l+1}(\sqrt{8 r})}{\sqrt{8 r}}+\sin \delta_{l} \frac{J_{2 l+1}(\sqrt{8 r})}{\sqrt{8 r}}$,

$$
\begin{aligned}
R_{l}^{0, \text { out }}(r) & =R_{l}^{0, \mathrm{reg}}(r)+i R_{l}^{0, \text { irreg }}(r) \\
& =e^{i \delta_{l}}\left(\frac{J_{2 l+1}(\sqrt{8 r})}{\sqrt{8 r}}+i \frac{Y_{2 l+1}(\sqrt{8 r})}{\sqrt{8 r}}\right) .
\end{aligned}
$$

$R_{l}^{0, \text { reg }}$ is the wave function that is regular at the origin, $R_{l}^{0 \text {,irreg }}$ is the one that is irregular at the origin, and $R_{l}^{0, \text { out }}$ is the outgoing wave function at large distances. $\delta_{l}$ is the phase shift to the Coulomb wave caused by the sodium core. $\delta_{l}=\mu_{l} / \pi$ is set by taking the quantum defect $\mu_{l}$ on the branch [5]

$$
-\frac{1}{2}<\mu_{l}<+\frac{1}{2}
$$

i.e.,

$$
\mu_{l} \rightarrow\left(\mu_{l}+\frac{1}{2}\right)(\bmod 1)-\frac{1}{2}
$$

This choice ensures the proper sign of the dipole matrix element $I_{\mathrm{Na}}\left(n, l, l^{\prime}\right)$. The values are

$$
\begin{aligned}
& \mu_{0}=1.350 \rightarrow+0.350, \\
& \mu_{1}=0.856 \rightarrow-0.144, \\
& \mu_{2}=0.014, \quad \mu_{l \geq 3} \approx 0 .
\end{aligned}
$$

The asymptotic forms for large $r$ (valid for $5 a_{0} \lesssim r \lesssim 50 a_{0}$ ) of these wave functions are

$$
\begin{array}{r}
R_{l}^{0, \mathrm{reg}}(r)=\left[\frac{2}{\pi \sqrt{8 r}}\right]^{1 / 2} \cos \left[\sqrt{8 r}-\left(l+\frac{1}{2}\right) \pi\right. \\
\left.-\pi / 4+\delta_{l}\right] / \sqrt{8 r}
\end{array}
$$




$$
\begin{array}{r}
R_{l}^{0, \text { irreg }}(r)=\left(\frac{2}{\pi \sqrt{8 r}}\right)^{1 / 2} \sin \left[\sqrt{8 r}-\left(l+\frac{1}{2}\right) \pi\right. \\
\left.-\pi / 4+\delta_{l}\right] / \sqrt{8 r}, \\
R_{l}^{0, \text { out }}(r)=\left(\frac{2}{\pi \sqrt{8 r}}\right)^{1 / 2} \exp \left\{i \left[\sqrt{8 r}-\left(l+\frac{1}{2}\right) \pi\right.\right. \\
\left.\left.-\pi / 4+\delta_{l}\right]\right\} / \sqrt{8 r} .
\end{array}
$$

Equations (4.2) are exact solutions to the Coulomb Schrödinger equation at $E=0$, and they give an adequate approximation to the solution for a reasonable band around $E=0$.

The ionization cross section is proportional to the dipole matrix elements between the initial states and the $E \approx 0$ states. Let us define

$$
I_{\mathrm{Na}}\left(n, l, l^{\prime}\right)=\sqrt{8} \int_{0}^{\infty} R_{n l}(r) R_{l^{\prime}}^{0, \mathrm{reg}}(r) r^{3} d r,
$$

where $R_{n l}(r)$ represents a radial factor for the initial state and $R_{l}^{0, \text { reg }}(r)$ is the regular solution at $E=0$, normalized so that its asymptotic part matches Eq. (4.3a). Such integrals are analogous to the hydrogenic integral [II, (4.12)], and for sodium, the integral $I_{\mathrm{Na}}\left(n, l, l^{\prime}\right)$ will replace the hydrogenic integral $I\left(n, l, l^{\prime}\right)$ everywhere in our formulas.

In comparison, Harmin, in his calculation, defined similar radial matrix elements, but normalized his wave functions differently. His wave functions were normalized by $\delta$ functions in energy

$\int_{0}^{\infty} R_{l \text { Harmin }}^{\text {reg }}(r, E) R_{l \text { Harmin }}^{\text {reg }}\left(r, E^{\prime}\right) r^{2} d r=\delta\left(E-E^{\prime}\right)$,

and a detailed analysis shows that our radial wave functions differ from his by

$$
R_{l}^{0, \text { reg }}(r)=\sqrt{\frac{1}{2}} R_{\text {Harmin }}^{\text {reg }}(r, E=0) .
$$

It follows that our radial integrals differ from his by the same factor. He gave values for the required integrals based upon an unpublished calculation by K. T. Cheng. We will use those values (divided by $\sqrt{2}$ ) in our calculation:

$$
\begin{aligned}
& I_{\mathrm{Na}}(3,1,0)=\sqrt{\frac{1}{2}}(3.32-5.39 E), \\
& I_{\mathrm{Na}}(3,1,2)=\sqrt{\frac{1}{2}}(5.79-152.2 E),
\end{aligned}
$$

where $E$ is in hartrees.

\section{THE SMOOTH-BACKGROUND ABSORPTION, THE OUTGOING WAVES, AND THE $\mathscr{y}$ 's}

With the radial wave functions defined above, the direct part of the Green's function is

$$
\begin{aligned}
& G_{\mathrm{dir}}^{+}\left(\mathbf{r}, \mathbf{r}^{\prime}, E\right)=\sum_{l, m} Y_{l m}(\theta, \varphi) g_{l}^{E}\left(r, r^{\prime}\right) Y_{l m}^{*}\left(\theta^{\prime}, \varphi^{\prime}\right) \\
& g_{l}^{E}\left(r, r^{\prime}\right)=\frac{2 R_{l}^{E, \operatorname{Reg}_{(}}\left(r_{<}\right) R_{l}^{E, \text { out }}\left(r_{>}\right)}{r^{\prime 2} W\left(R_{l}^{E, \operatorname{Reg}_{(}}\left(r^{\prime}\right) R_{l}^{E, \text { out }}\left(r^{\prime}\right)\right)}
\end{aligned}
$$

where $W$ is the Wronskian

$$
W=R_{1} R_{2}^{\prime}-R_{2} R_{1}^{\prime}=i /\left(8 \pi r^{\prime 2}\right) .
$$

This formula applies to hydrogen or to sodium. In the latter case, the Green's function is understood to contain a unit operator acting upon spin states.

\section{A. Background absorption}

As was shown in Ref. [2], the smooth-background absorption is related to the direct part of the Green's function by the formula

$D f_{0}(E \approx 0)=-\frac{2\left(E-E_{i}\right)}{\pi}\left\langle D \psi_{i}\left|\operatorname{Im} \hat{G}_{\mathrm{dir}}^{+}\right| D \psi_{i}\right\rangle$.

Straightforward application of Eq. (5.1) and the appropriate formula for $D \psi_{i}$ leads to the following results.

For hydrogen

$$
\begin{aligned}
D f_{0}(E \approx 0) & =-4\left(E-E_{i}\right) \sum_{l^{\prime}, l}\left|d_{l} b_{l^{\prime} m}^{l} I\left(n, l, l^{\prime}\right)\right|^{2} \\
& =\frac{1}{4}\left[\frac{1}{3} I(2,0,1)^{2}+\frac{4}{15} I(2,1,2)^{2}+\frac{1}{3} I(2,1,0)^{2}\right] \\
& =3.939 .
\end{aligned}
$$

This value applies to either initial state $(2 s \pm 2 p)$.

For pseudohydrogen,

$$
\begin{aligned}
D f_{0}(E \approx 0) & =-4\left(E-E_{i}\right) \sum_{l^{\prime}}\left|b_{l^{\prime} m}^{l} I\left(n, l, l^{\prime}\right)\right|^{2} \\
& =\frac{2}{9}\left[\frac{1}{3} I(3,1,0)^{2}+\frac{4}{15} I(3,1,2)^{2}\right] \\
& =8.356 .
\end{aligned}
$$

For sodium,

$D f_{0}(E \approx 0)=-4\left(E-E_{i}\right) \sum_{l^{\prime}, m}\left|C_{m}^{j} b_{l^{\prime} m}^{l^{\prime}} I_{\mathrm{Na}}\left(n, l, l^{\prime}\right)\right|^{2}$.

In this case, the values for the two possible initial states are not the same. For $j=\frac{3}{2}, m_{j}=\frac{1}{2}$,

$$
\begin{aligned}
D f_{0}(E \approx 0) & =\frac{2}{3} D f_{0}(E \approx 0, m=0 \rightarrow 0)+\frac{1}{3} D f_{0}(E \approx 0, m=1 \rightarrow 1) \\
& =4 E_{i}\left[\frac{2}{9} I_{\mathrm{Na}}(3,1,0)^{2}+\frac{8}{45} I_{\mathrm{Na}}(3,1,2)^{2}+\frac{1}{15} I_{\mathrm{Na}}(3,1,2)^{2}\right] \\
& =2.373
\end{aligned}
$$

while for $j=\frac{1}{2}, m_{j}=\frac{1}{2}$, 


$$
\begin{aligned}
D f_{0}(E \approx 0) & =\frac{1}{3} D f_{0}(E \approx 0, m=0 \rightarrow 0)+\frac{2}{3} D f_{0}(E \approx 0, m=1 \rightarrow 1) \\
& =4 E_{i}\left[\frac{1}{9} I_{\mathrm{Na}}(3,1,0)^{2}+\frac{4}{45} I_{\mathrm{Na}}(3,1,2)^{2}+\frac{2}{15} I_{\mathrm{Na}}(3,1,2)^{2}\right] \\
& =1.936 .
\end{aligned}
$$

Therefore, $l$-s coupling simply superposes the background absorption with different values of $m$.

\section{B. Outgoing waves}

The quantity $G_{\text {dir }}^{+}\left|D \psi_{i}\right\rangle$ represents the initial outgoing wave that is produced when the photon ionizes the atom. For $r \gtrsim 5 a_{0}$, the asymptotic expression can be used for $R_{l}^{E=0, \text { out }}\left(r_{>}\right)$, and the outgoing wave becomes

$$
\begin{aligned}
\psi_{\text {out }}(\mathbf{r}) & =G_{\text {dir }}^{+}\left|D \psi_{i}\right\rangle \\
& =\sum_{m}(-i) \pi^{1 / 2} 2^{3 / 4} r^{-3 / 4} e^{-i 3 \pi / 4} e^{i \sqrt{8 r}} y_{m}(\theta) e^{i m \varphi} \\
& =\sum_{m} \psi_{\text {out }}^{m}(r, \theta) e^{i m \varphi},
\end{aligned}
$$

in which

$$
\begin{aligned}
\mathscr{y}_{m}(\theta)=\sum_{l}(-1)^{l} Y_{l m}(\theta, 0) e^{i \delta_{l}} \int & \sqrt{8} Y_{l m}^{*}\left(\theta^{\prime} \varphi^{\prime}\right) R_{l}^{0, \mathrm{reg}}\left(r^{\prime}\right) \\
& \times D \psi_{i}\left(r^{\prime}\right) r^{\prime 3} d \mathbf{r}^{\prime}
\end{aligned}
$$

The functions $\mathscr{y}_{m}(\theta)$ represent the angular distribution of the outgoing waves. They are evaluated for the various cases by again using the appropriate initial state in Eq. (5.10).

For hydrogen, we obtain

$$
\mathscr{y}_{m}(\theta)=\sum_{l, l^{\prime}} d_{l}(-1)^{l^{\prime}} I\left(n, l, l^{\prime}\right) b_{l^{\prime} m}^{l} Y_{l^{\prime} m}(\theta, 0)
$$

or, explicitly,

$$
\begin{aligned}
\mathscr{F}_{0}(\theta)=\sqrt{\frac{1}{2}}[ & \lambda I(2,1,0) b_{00}^{1} Y_{00}(\theta, 0) \\
& +\lambda I(2,1,2) b_{20}^{1} Y_{20}(\theta, 0) \\
& \left.-I(2,0,1) b_{10}^{0} Y_{10}(\theta, 0)\right] .
\end{aligned}
$$

For the special case $\theta=0$,

$$
\begin{aligned}
y_{0}(0)= & (\lambda / \sqrt{24 \pi}) I(2,1,0)+(\lambda / \sqrt{16 \pi}) I(2,1,2) \\
& -(1 / \sqrt{8 \pi}) I(2,0,1) .
\end{aligned}
$$

The value of $y_{0}(0)$ is -2.3382 for $\lambda=-1$, and 0.4676 for $\lambda=1$.

For pseudohydrogen, Eq. (2.6) is correct, and

$$
\mathscr{y}_{0}(\theta)=b_{00}^{1} I(3,1,0) Y_{00}(\theta, 0)+b_{20}^{1} I(3,1,2) Y_{20}(\theta, 0) .
$$

All the other $y$ 's are equal to zero. The value at $\theta=0$ is

$$
\begin{aligned}
\mathscr{y}_{0}(0) & =\sqrt{1 / 12 \pi} I(3,1,0)+\sqrt{1 / 3 \pi} I(3,1,2) \\
& =4.2290
\end{aligned}
$$

For sodium, due to the spin-orbit coupling, for each quantum number $j$, the outgoing wave is a spinor function-i.e., the angular distribution of outgoing waves depends on whether the electron spin is up or down. The general formula for the $y_{m}(\theta)$ is

$\mathscr{y}_{m}(\theta)=\sum_{l^{\prime}} C_{m}^{j} \chi_{m_{j}-m}(-1)^{l^{\prime}} I\left(n, l, l^{\prime}\right) b_{l^{\prime} m}^{l} Y_{l^{\prime} m}(\theta, 0) e^{i \delta_{l^{\prime}}}$

The specific forms with different quantum numbers are, for $j=\frac{3}{2}, m_{j}=\frac{1}{2}$,

$$
\begin{gathered}
\begin{array}{c}
\mathscr{y}_{0}(\theta)=\sqrt{\frac{2}{3}}\left[b_{00}^{1} I_{\mathrm{Na}}(3,1,0) Y_{00}(\theta, 0) e^{i \delta_{0}}\right. \\
\left.+b_{20}^{1} I_{\mathrm{Na}}(3,1,2) Y_{20}(\theta, 0) e^{i \delta_{2}}\right] \alpha,
\end{array} \\
\begin{array}{c}
y_{1}(\theta)=\sqrt{\frac{1}{3}} b_{21}^{1} I_{\mathrm{Na}}(3,1,2) Y_{21}(\theta, 0) e^{i \delta_{2}} \beta \\
\text { and, for } j=\frac{1}{2}, m_{j}=\frac{1}{2},
\end{array} \\
\begin{array}{c}
\mathscr{y}_{0}(\theta)=\sqrt{\frac{1}{3}}\left[b_{00}^{1} I_{\mathrm{Na}}(3,1,0) Y_{00}(\theta, 0) e^{i \delta_{0}}\right. \\
\left.\quad+b_{20}^{1} I_{\mathrm{Na}}(3,1,2) Y_{20}(\theta, 0) e^{i \delta_{2}}\right] \alpha,
\end{array} \\
\mathscr{y}_{1}(\theta)=-\sqrt{\frac{2}{3}} b_{21}^{1} I_{\mathrm{Na}}(3,1,2) Y_{21}(\theta, 0) e^{i \delta_{2}} \beta .
\end{gathered}
$$

When $\theta=0$, both $y$ 's only have a spin-up part. In other words, all our observed oscillations in sodium are caused by the $m_{f}=0$ final state:

$$
\begin{aligned}
y_{0}(0) & =\frac{1}{3 \sqrt{2 \pi}}\left[I_{\mathrm{Na}}(3,1,0) e^{i \delta_{0}}+2 I_{\mathrm{Na}}(3,1,2) e^{i \delta_{2}}\right] \alpha \\
& =\left(0.312 e^{i \delta_{0}}+1.09 e^{i \delta_{2}}\right) \alpha, \quad j=\frac{3}{2}, m_{j}=\frac{1}{2} \\
y_{0}(0) & =\frac{1}{3 \sqrt{4 \pi}}\left[I_{\mathrm{Na}}(3,1,0) e^{i \delta_{0}}+2 I_{\mathrm{Na}}(3,1,2) e^{i \delta_{2}}\right] \alpha \\
& =\sqrt{\frac{1}{2}}\left(0.312 e^{i \delta_{0}}+1.09 e^{i \delta_{2}}\right) \alpha, \quad j=\frac{1}{2}, m_{j}=\frac{1}{2}
\end{aligned}
$$

The angular distributions of the outgoing waves are plotted in Fig. 1. 


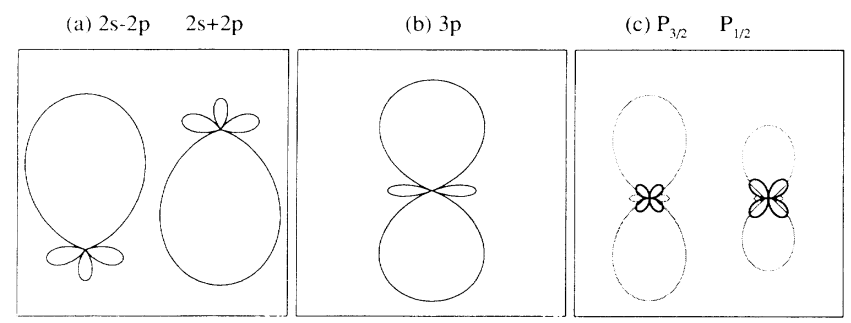

FIG. 1. The angular distribution of the outgoing waves: (a) $2 s \pm 2 p$ initial state; (b) $3 p$ initial state; (c) $3{ }^{2} P_{3 / 2,1 / 2}$ initial state. Dashed lines are spin-up states, and solid lines are spin-down states.

\section{SEMICLASSICAL RETURNING WAVES}

As is well known, the semiclassical wave function can be calculated from classical trajectories provided the wave function is known on an initial surface. Since our Hamiltonian has cylindrical symmetry, the threedimensional wave function can be separated:

$$
\begin{aligned}
\psi(r, \theta, \varphi)= & \psi_{\text {out }}^{m}\left(r_{i}, \theta_{i}\right) A(r, \theta, \varphi) \\
& \times \exp \left\{i\left[S_{2}(r, \theta) / \hbar-\mu \pi / 2\right]\right\} e^{i m \varphi} .
\end{aligned}
$$

We take the boundary sphere as the initial surface. The amplitude is related to a two-dimensional quantity $A_{2}(r, \theta)$ by the formula

$$
A(r, \theta, \varphi)=A_{2}(r, \theta)\left|r_{i}^{2} \sin \theta_{i} / r^{2} \sin \theta\right|^{1 / 2} .
$$

In Ref. [2] which dealt with orbits of energy $E \approx 0$ in a magnetic field, all the orbits were unstable. Therefore, each closed orbit contributed a substantial oscillation only on its first return, and in most cases, the contribution of the second or third returns of an orbit were negligible. However, in the case of an electric field, for $E<0$ the relevant orbits are stable, and for $E>0$ the instability is rather weak. Therefore, many repetitions of a given orbit can produce visible effects. For the hydrogen atom, the effects can be calculated using only minor modifications of Eq: (2.5). For sodium, on the other hand, the effects of repetitions are nontrivial.

\section{A. The amplitude $A_{2}$}

The amplitude is calculated by computing each classical closed orbit and its neighbors, and evaluating the two-dimensional Jacobian

$$
J_{2}\left(t, \theta_{i}\right)=\frac{\partial(r, \theta)}{\partial\left(t, \theta_{i}\right)}
$$

at the initial and final boundary $r_{b}$; then,

$$
A_{2}^{1}=\left|J_{2}\left(0, \theta_{i}\right) / J_{2}\left(t_{1}, \theta_{i}\right)\right|^{1 / 2},
$$

where $t_{1}$ is the time of first return to the boundary.

When a closed orbit returns to the nucleus, the Coulomb field sends the electron back to retrace exactly the orbit in reverse. However, the neighbors do not retrace their steps. They pass behind the nucleus and go back out close to the closed orbit, following it around until it returns again. The amplitude for the $n$th return is obtained by evaluating the same two-dimensional Jacobian (6.3) at $t_{n}$, the time of the $n$th return to the boundary,

$$
A_{2}^{n}=\left|J_{2}\left(0, \theta_{i}\right) / J_{2}\left(t_{n}, \theta_{i}\right)\right|^{1 / 2} \text {. }
$$

Computationally, instead of integrating the neighboring orbits step by step when they are close to the nucleus, we neglect the electric field inside the boundary radius and use analytical formulas for Kepler orbits. Specifically, on each return of a trajectory to the boundary $r_{b}$, the value of $\theta$ and $p_{\theta}$ are recorded; then, using standard formulas for Kepler orbits, one can show that the trajectory goes back out through the boundary at an angle

$$
\theta^{\prime}=\theta-2\left(\operatorname{sgn} p_{\theta}\right) \sin ^{-1}\left[\frac{p_{\theta}^{2} / r_{b}-1}{\left(1+2 E p_{\theta}^{2}\right)^{1 / 2}}+\pi / 2\right]
$$

and with $p_{\theta}^{\prime}=p_{\theta}$. Hence, the neighbor is launched in a new direction, and it can be followed numerically until its next return. (A better way to treat this problem is given by Mao and Delos [12].)

\section{B. The phase}

The phase of the semiclassical wave function is calculated as follows. In Eq. (2.5) the phase $S_{2}^{k}$ is $\int \mathrm{p} \cdot d \mathbf{q}$ on a closed orbit starting and ending at the boundary $r_{b}$, and the term $2\left(8 r_{b}\right)^{1 / 2}$ corrects this phase to include the action integral from $r=0$ to $r=r_{b}$. Hence, each time the orbit passes through the nucleus, the phase increase by $2\left(8 r_{b}\right)^{1 / 2}$. It follows that the semiclassical phase for the $n$th return of the $k$ th closed orbit is

$$
S^{k, n}=n\left(S_{2}^{k}+2 \sqrt{8 r_{b}}\right) .
$$

If $E \neq 0$, then $\left(8 r_{b}\right)^{1 / 2}$ should be replaced by

$$
\int_{0}^{r_{b}} \sqrt{2 E+2 / r} d r=\left\{\begin{array}{l}
r\left[2\left(E+\frac{1}{r}\right)\right]^{1 / 2}-\left(\frac{2}{-E}\right)^{1 / 2}\left[\arctan \left(-1-\frac{1}{E r}\right)^{1 / 2}-\frac{\pi}{2}\right], \quad E<0 \\
r\left[2\left[E+\frac{1}{r}\right)\right]^{1 / 2}+\left(\frac{2}{E}\right)^{1 / 2} \ln (\sqrt{E r}+\sqrt{E r+1}), \quad E>0 .
\end{array}\right.
$$


The Maslov index is computed by counting caustics encountered by the trajectory [8]. For any Hamiltonian of the form

$$
H=p^{2} / 2 m+V(\mathbf{q}),
$$

the Maslov index increases by 1 at each simple zero of the Jacobian $J(t)$. As trajectories are integrated, we monitor $J_{2}(t)$ for changes of sign, and increment $\mu$ at each such change. In addition, as stated in Ref. [2], the index increases by 1 each time an orbit passes through $\rho=0$. (One might expect that the Maslov index for the $n$th repetition of the orbit, $\mu^{n}$, is equal to $n \mu^{1}$; however, this relation does not always hold. A general formula for $\mu^{n}$ is given in Ref. [12].)

Furthermore, at each return to the nucleus the Maslov index increases by 2 if $\theta_{f} \neq 0$ and by 1 if $\theta_{f}=0$. This change is due to a focusing effect of the orbits close to the nucleus.

For $\theta_{f} \neq 0$, the proof is a trivial extension of formulas given in II. Using the approximation discussed therein, we derived a closed-form expression for the incoming part of the returning wave [II, (4.23a)]:

$$
\begin{aligned}
\left(\psi_{c, \theta_{f}}\right)_{\text {inc }}= & (-1)^{m} e^{i \pi / 2} 2^{-3 / 2} \pi^{-1} \\
& \times \exp \left\{-i 2 \sqrt{r\left[1+\cos \left(\theta-\theta_{f}\right)\right]}\right\} \\
& \times\left(r \sin \theta_{f} \sin \theta\right)^{-1 / 2} e^{i m \varphi} .
\end{aligned}
$$

By precisely the same method, the corresponding expression for the outgoing part of the returning wave is

$$
\begin{aligned}
\left(\psi_{c, \theta_{f}}\right)_{\text {scat }}= & (-1)^{m} e^{-i \pi / 2} 2^{-3 / 2} \pi^{-1} \\
& \times \exp \left\{i 2 \sqrt{r\left[1+\cos \left(\theta-\theta_{f}\right)\right]}\right\} \\
& \times\left(r \sin \theta_{f} \sin \theta\right)^{-1 / 2} e^{i m \varphi}
\end{aligned}
$$

These asymptotic expressions hold for $r \gtrsim 10 a_{0}$ and $\theta$ close to $\theta_{f}$. Hence, at the boundary $r=r_{b}, \theta=\theta_{f}$, the scattered wave is related to the incoming wave by

$$
\left(\psi_{c, \theta_{f}}\right)_{\text {scat }}=e^{-i \pi} e^{i 2 \sqrt{8 r_{b}}}\left(\psi_{c, \theta_{f}}\right)_{\text {inc }}
$$

Clearly, the phase difference between the Coulomb scattered and the incoming wave is $\left(2 \sqrt{8 r_{b}}-\pi\right)$. The first term is the classical action from the boundary to the origin and back to the boundary. The second term is naturally incorporated into the Maslov index for the trajectory, and it shows that this index increases by 2 in each return to the nucleus. (The case $\theta=0$ will be examined in Sec. VIII.)

\section{RETURNING AND SCATTERED WAVES CLOSE TO THE NUCLEUS (FOR $\theta \neq 0$ )}

As stated in Ref. [2], the returning waves are assumed to be similar to the wave that would be obtained from a plane-wave source at infinite distance in a pure Coulomb field. According to the description given in Fig. 3 of Sec. IV D of Ref. [2], we took a zero-energy Coulomb scattering wave, rotated it so that its incoming direction would correspond to the direction of approach of the $k \mathrm{th}$ trajectory, and then spun it around the zero axis to recover cylindrical symmetry.

For hydrogen, the resulting approximations for the returning waves, and their effect on the oscillator-strength density, were fully described in II. Here we develop the corresponding approximation for alkali-metal atoms, incorporating the phase shifts due to the atomic core.

Waves returning to the sodium ion must satisfy two conditions: (i) They must be the solutions of the Schrödinger equation for $\mathrm{Na}$, and (ii) since the potential deviates from Coulomb form only at small $r$, the incoming waves must be same as hydrogenic ones,

$$
\left(\psi_{\mathrm{Na}, \theta_{f}}^{m}\right)_{\mathrm{inc}}=\left(\psi_{c, \theta_{f}}^{m}\right)_{\mathrm{inc}} .
$$

The required expressions for the incoming part of the wave functions are as indicated in [II, (4.23a) and (4.23b)]:

$$
\begin{aligned}
&\left(\psi_{\mathrm{Na}, \theta_{f}}^{m}\right)_{\mathrm{inc}}=(-1)^{m} e^{i \pi / 2} 2^{-3 / 2} \pi^{-1} \\
&\left.\times \exp \left\{-i 2 \sqrt{r\left[1+\cos \left(\theta-\theta_{f}\right)\right.}\right]\right\} \\
& \times\left(r \sin \theta_{f} \sin \theta\right)^{-1 / 2} e^{i m \varphi} \\
&\left(\psi_{\mathrm{Na}, \theta_{f}}^{m}\right)_{\mathrm{inc}} \\
&=\frac{4 \pi}{\sqrt{2}} \sum_{l}(-1)^{l-m} 2^{-5 / 4} \pi^{-1 / 2} Y_{l m}^{*}\left(\theta_{f}, 0\right) Y_{l m}(\theta, \varphi) \\
& \times \exp [i(-\sqrt{8 r}+l \pi+3 \pi / 4)] / r^{3 / 4}
\end{aligned}
$$

The partial-wave expansion of the returning wave is

$$
\psi_{\mathrm{Na}, \theta_{f}}^{m}=\sum_{l} A_{l} R_{l}^{0, \mathrm{reg}}(r) Y_{l m}(\theta, \varphi),
$$

where $R_{l}^{0, \text { reg }}(r)$ is given in Eq. (4.3a). Here is the point at which waves returning to a sodium ion differ from those returning to a hydrogen ion - the radial wave functions are different. If we use Eq. (4.3a) to separate $\psi_{\mathrm{Na}, \theta_{f}}^{m}$ into incoming and outgoing waves and match the incoming part with (7.2), then

$$
A_{l}=e^{i \delta_{l}} \frac{4 \pi}{\sqrt{2}}(-1)^{l-m} Y_{l m}^{*}\left(\theta_{f}, 0\right) \sqrt{8} ;
$$

therefore,

$$
\begin{aligned}
\psi_{\mathrm{Na}, \theta_{f}}^{m}=\frac{4 \pi}{\sqrt{2}} \sum_{l}(-1)^{l-m} Y_{l m}^{*}\left(\theta_{f}, 0\right) Y_{l m}(\theta, \varphi) \sqrt{8} \\
\times R_{l}^{0, \mathrm{reg}}(r) e^{i \delta_{l}} .
\end{aligned}
$$

Let us now evaluate the overlap of the returning wave with the "source" $\left\langle D \psi_{i} \mid \psi_{\mathrm{Na}, \theta_{f}}^{m}\right\rangle$ [noting also that $Y_{l m}\left(\theta_{f}, 0\right)$ is real ]: 


$$
\begin{aligned}
(-1)^{m}\left\langle D \psi_{i} \mid \psi_{\mathrm{Na}, \theta_{f}}^{m}\right\rangle & =\frac{4 \pi}{\sqrt{2}} \sum_{l}(-1)^{l} Y_{l m}^{*}\left(\theta_{f}, 0\right) e^{i \delta_{l}}\left\langle D \psi_{i} \mid \sqrt{8} R_{l}^{0, \mathrm{reg}} Y_{l m}\right\rangle \\
& =\frac{4 \pi}{\sqrt{2}} \sum_{l}(-1)^{l} e^{i \delta_{l}}\left[Y_{l m}\left(\theta_{f}, 0\right) C_{m}^{j} I\left(n, l, l^{\prime}\right) b_{l^{\prime} m}^{l} \chi_{m_{j}-m}\right]^{\dagger} \\
& =\frac{4 \pi}{\sqrt{2}} \widetilde{\mathscr{Y}}_{m}\left(\theta_{f}\right) .
\end{aligned}
$$

$\tilde{\mathscr{y}}_{m}(\theta)$ is the "unexpected conjugate" of $\mathscr{y}_{m}(\theta)$ : It contains the Hermitian conjugate of the spin state $\chi_{m_{j}-m}^{\dagger}$, and (in principle) complex conjugates of the coefficients $b_{l^{\prime} m}^{l}, C_{m}^{j}$, the integral $I\left(n, l, l^{\prime}\right)$, and $Y_{l m}\left(\theta_{f}, 0\right)$, but it does not contain the complex conjugates of $e^{i \delta_{l}}$. In our case (linear polarization), all the quantities marked [ ] $]^{\dagger}$ are real, so $\tilde{\mathscr{y}}_{m}(\theta)$ is the transpose spinor to $\mathscr{y}_{m}(\theta)$.

Each returning orbit carries with it a returning wave which is equal to a constant times $\psi_{\mathrm{Na}, \theta_{f}}^{m}$ defined above:

$$
\psi_{\text {ret }}^{m}(r, \theta, \varphi)=e^{i m \varphi} A_{2}^{m}(r, \theta) \exp \left\{i\left[S_{2}^{m}(r, \theta)-\mu^{m} \pi / 2\right]\right\}\left(r_{i}^{2} \sin \theta_{i} / r^{2} \sin \theta\right)^{1 / 2} \psi_{\text {out }}^{m}\left(r_{i}, \theta_{i}\right) .
$$

This is set equal to a constant times (7.1) on a boundary point $r=r_{f}, \theta=\theta_{f}$ :

$$
\psi_{\mathrm{ret}}^{m}\left(r_{f}, \theta_{f}, \varphi\right)=N_{m}\left(\psi_{\mathrm{Na}, \theta_{f}}^{m}\left(r_{f}, \theta_{f}, \varphi\right)\right)_{\mathrm{inc}} .
$$

Combining Eqs. (7.1) and (7.8), we get

$$
N_{m}=A_{2}^{m}\left(r_{f}, \theta_{f}\right) \exp \left\{i\left[S_{2}^{m}\left(r_{f}, \theta_{f}\right)-\mu^{m} \pi / 2\right]\right\} \psi_{\text {out }}^{m}\left(r_{i}, \theta_{i}\right) e^{-i \pi / 2} 2^{3 / 2} \pi e^{i \sqrt{8 r_{i}}}\left(r_{i} / r_{f}^{1 / 2}\right)\left(\sin \theta_{i} \sin \theta_{f}\right)^{1 / 2}(-1)^{m} .
$$

Setting $r_{i}=r_{f}=r_{b}$ and using Eq. (5.8) for $\psi_{\text {out }}^{m}$, we obtain

$$
N_{m}\left\langle D \psi_{i} \mid \psi_{\mathrm{Na}, \theta_{f}}^{m}\right\rangle=(-1) 2^{15 / 4} \pi^{5 / 2} r_{b}^{-1 / 4} e^{i 2 \sqrt{8 r_{b}}}\left(\sin \theta_{i} \sin \theta_{f}\right)^{1 / 2} e^{-i 3 \pi / 4} A_{2}^{m} \exp \left\{i\left[S_{2}^{m}(r, \theta)-\mu^{m} \pi / 2\right]\right\} \mathscr{y}_{m}\left(\theta_{i}\right) \widetilde{\mathscr{y}}_{m}\left(\theta_{f}\right) .
$$

Near the boundary, the scattered part of the wave function can be extracted from Eq. (7.5):

$$
\left(\psi_{\mathrm{Na}, \theta_{f}}^{m}\right)_{\text {scat }}=\frac{4 \pi}{\sqrt{2}} \sum_{l \geq|m|}(-1)^{l-m} 2^{-5 / 4} \pi^{-1 / 2} Y_{l m}^{*}\left(\theta_{f}, 0\right) Y_{l m}(\theta, \varphi) r^{-3 / 4} \exp \left\{i\left[(8 r)^{1 / 2}-l \pi-3 \pi / 4\right]\right\} e^{i 2 \delta_{l}} .
$$

It is obvious that $\left(\psi_{\mathrm{Na}, \theta_{f}}^{m}\right)_{\text {scat }}$ can be separated into two parts [9],

$$
\left(\psi_{\mathrm{Na}, \theta_{f}}^{m}\right)_{\text {scat }}=\left(\psi_{c, \theta_{f}}^{m}\right)_{\text {scat }}+\left(\psi_{\text {core }, \theta_{f}}^{m}\right)_{\text {scat }},
$$

where

$$
\left(\psi_{c, \theta_{f}}^{m}\right)_{\text {scat }}=\frac{4 \pi}{\sqrt{2}} \sum_{l \geq|m|}(-1)^{l-m} 2^{-5 / 4} \pi^{-1 / 2} Y_{l m}^{*}\left(\theta_{f}, 0\right) Y_{l m}(\theta, \varphi) r^{-3 / 4} \exp \left\{i\left[(8 r)^{1 / 2}-l \pi-3 \pi / 4\right]\right\}
$$

and

$$
\left(\psi_{\text {core }, \theta_{f}}^{m}\right)_{\text {scat }}=\frac{4 \pi}{\sqrt{2}} \sum_{l \geq|m|}(-1)^{l-m} 2^{-5 / 4} \pi^{-1 / 2} Y_{l m}^{*}\left(\theta_{f}, 0\right) Y_{l m}(\theta, \varphi) r^{-3 / 4} \exp \left\{i\left[(8 r)^{1 / 2}-l \pi-3 \pi / 4\right]\right\}\left(e^{i 2 \delta_{l}}-1\right) .
$$

$\left(\psi_{c, \theta_{f}}^{m}\right)_{\text {scat }}$ is the cylindrically modified Coulomb-scattered wave; it is the same as the scattered wave that arises in hydrogen. These waves are strongly backward focused, so they go out in the $\theta_{f}$ direction. These waves then retrace the closed orbit in reverse, and return to the nucleus later to produce oscillations that we associate with repetitions of the orbit. $\left(\psi_{\mathrm{core}, \theta_{f}}^{m}\right)_{\text {scat }}$ is the wave scattered from the very compact core of the sodium ion. Since for sodium the phase shifts $\delta_{l}$ decrease quickly to zero with increasing $l$, the partial-wave expansion of the core-scattered waves contains only three terms $(l=0,1,2)$, with $s$ and $p$ waves strongly dominant. In contrast to the Coulomb-scattering wave, which is strongly backward focused, the core-scattered wave is almost spherically symmetric. The core scatters the returning waves from each closed orbit and produces outgoing waves on every other closed orbit. Later, when these waves return, they give interference structure in the oscillator-strength density, and they again produce Coulomb-scattered and core-scattered waves (Fig. 2).

Our calculations show that the effect of core-scattered waves is small. Neglecting these terms, we form our final formula for the oscillator-strength density for $\theta_{f} \neq 0$ :

$$
D f_{1}(E)=\left[-2\left(E-E_{i}\right) / \pi\right] \operatorname{Im} \sum_{m, k_{m}, n} N_{m}\left\langle D \psi_{i} \mid \psi_{\mathrm{Na}, \theta_{f}}^{m}\right\rangle
$$




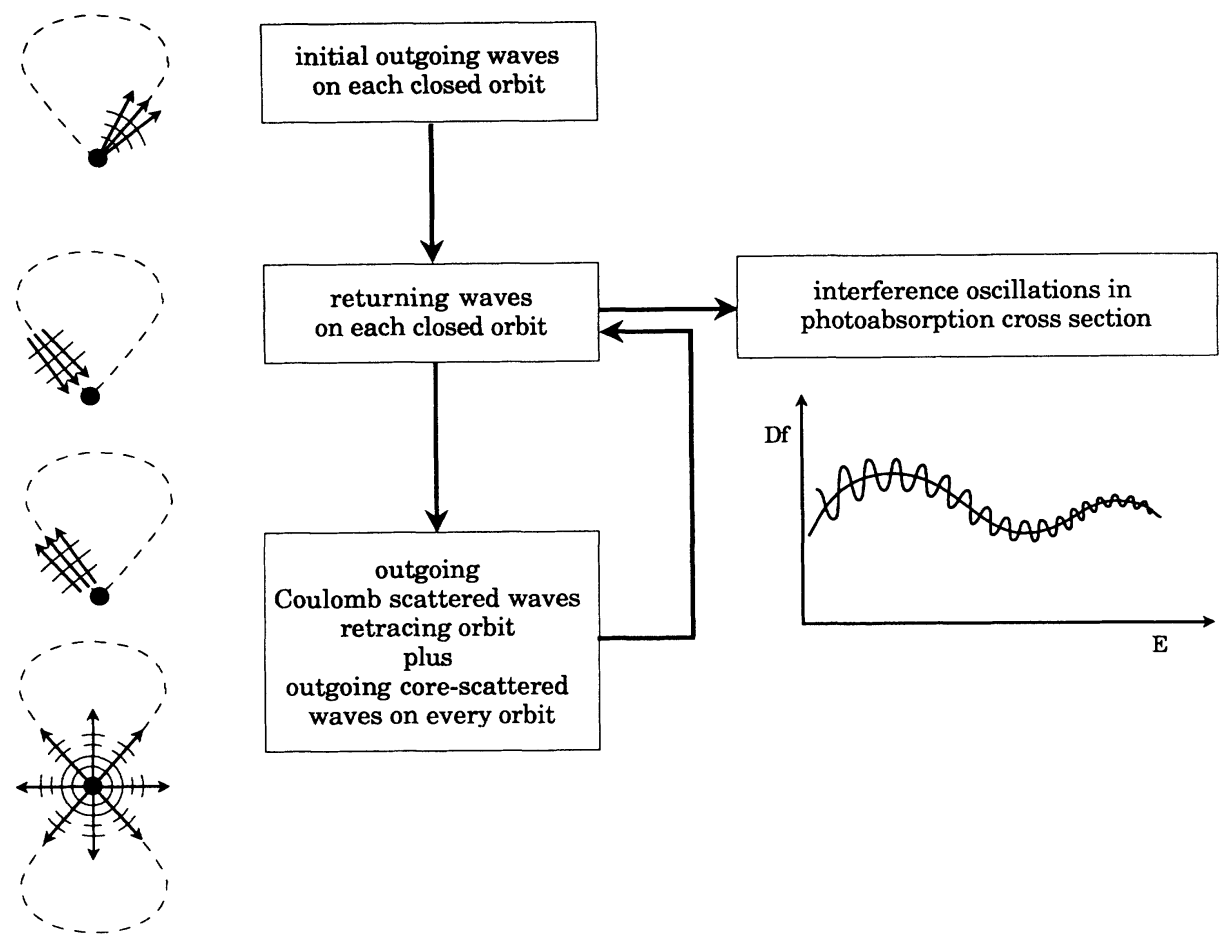

FIG. 2. Coulomb-scattered wave and core-scattered wave in the repetitions of the closed orbit.

$$
=\sum_{m, k_{m}, n} \operatorname{Im}\left[C_{m, k_{m}, n} \exp \left(i \Delta_{m, k_{m}, n}\right)\right]
$$

where

$$
\begin{aligned}
C_{m, k_{m}, n} \exp \left(i \Delta_{m, k_{m}, n}\right)= & \left(E-E_{i}\right) 2^{19 / 4} \pi^{3 / 2} r_{b}^{-1 / 2} e^{2 i\left(8 r_{b}\right)^{1 / 2}} e^{-i(3 / 4) \pi} \\
& \times\left(\sin \theta_{i}^{m, k_{m}} \sin \theta_{f}^{m, k_{m}}\right)^{1 / 2} A_{2}^{m, k_{m}, n} \exp \left[i\left(S^{m, k_{m}, n}-\frac{1}{2} \pi \mu^{m, k_{m}, n}\right)\right] \widetilde{\mathscr{Y}}_{m}\left(\theta_{f}^{m, k_{m}}\right) y_{m}\left(\theta_{i}^{m, k_{m}}\right) .
\end{aligned}
$$

The indices $m, k_{m}, n$ correspond to the $n$th repetition of the $k$ th closed orbit having $l_{z}=m \hbar$.

Equation (7.16) is a general formula for the oscillatorstrength density for hydrogen and pseudohydrogen, as well as sodium. The only difference among them is in the $\mathscr{y}_{m}(\theta)$ factors.

\section{THE SPECIAL CASE $\theta=0$}

If $m=0$, there is a closed orbit lying on the $z$ axis with $\theta_{i}=\theta_{f}=0$. For this orbit, the partial-wave expansions (7.2)-(7.9) [II, (4.22c)], and [II, (4.23b)] still apply. However, the closed-form expressions [II, (4.23a)] and (6.10) do not hold, since the required stationary-phase approximation does not apply for $\theta_{f}=0$. Therefore, the oscillator-strength density cannot be obtained from the general formula simply by taking the limit of $\theta_{f}=0$. This case must be analyzed separately.

\section{A. Returning and scattered waves}

If the electrons at large distance approach the nucleus along the positive $z$ direction, then the closed-form wave function is

$$
\psi \rightarrow \psi_{c, \theta_{f}=0}=J_{0}(2 \sqrt{\eta}),
$$

where $\eta=r+z=2 z$. Using the asymptotic approximation for the Bessel function, the closed-form expressions for the incoming part and the scattered part of the returning wave are

$$
\begin{aligned}
& \left(\psi_{c, \theta_{f}=0}\right)_{\mathrm{inc}}=\frac{1}{2}\left(\frac{2}{\pi \sqrt{8 z}}\right)^{1 / 2} e^{-i\left[(8 z)^{1 / 2}-\pi / 4\right]}, \\
& \left(\psi_{c, \theta_{f}=0}\right)_{\mathrm{scat}}=\frac{1}{2}\left(\frac{2}{\pi \sqrt{8 z}}\right)^{1 / 2} e^{i\left[(8 z)^{1 / 2}-\pi / 4\right]},
\end{aligned}
$$

so at the boundary $r=r_{b}$,

$$
\left(\psi_{c, \theta_{f}=0}\right)_{\mathrm{scat}}=e^{i(2 \sqrt{8 r}-\pi / 2)}\left(\psi_{c, \theta_{f}=0}\right)_{\mathrm{inc}} \text {. }
$$

This proves that for $\theta=0$, each time the orbit passes through the core, the Maslov index increases by 1 , and an additional phase $2 \sqrt{8 r}$ is added to the wave function.

If we consider sodium instead of hydrogen, then the in- 
coming waves remain the same as those for hydrogen, and as before, the scattered wave function is a combination of a Coulomb-scattered wave (8.3), which is focused on the $z$ axis, and a core-scattered wave, which is again ciescribed by (7.14b) with $\theta_{f}=0$. Writing the scattered wave in terms of $P_{l}(\cos \theta)$ instead of $Y_{l m}(\theta, \varphi)$, we obtain

$$
\begin{aligned}
\left(\psi_{\mathrm{Na}, \theta_{f}=0}^{m}\right)_{\text {scat }}= & \left(\psi_{c, \theta_{f}=0}^{m}\right)_{\text {scat }}+\left(\psi_{\text {core }, \theta_{f}=0}^{m}\right)_{\text {scat }}, \\
\left(\psi_{\text {core }, \theta_{f}=0}\right)_{\text {scat }}= & \frac{1}{2}\left[\frac{2}{\pi \sqrt{8 z}}\right]^{1 / 2} e^{i(\sqrt{8 r}-3 \pi / 4)} / r^{3 / 4} \\
& \times \sum_{l} \frac{2 l+1}{\sqrt{2}} P_{l}(\cos \theta)\left(e^{i 2 \delta_{l}}-1\right) .
\end{aligned}
$$

Again the core-scattered waves go out on every closed orbit and produce interference structure when they return (negligible in the cases we have examined).

\section{B. Formula for the oscillator-strength density $(\theta=0)$}

Only a few changes need to be made in order to derive the oscillator-strength density. (i) The amplitude of the semiclassical wave function [Eq. (6.2)] is undefined when $\theta=\theta_{i}=0$, so we need to take this limit carefully. Since the two-dimensional amplitude is

$$
A_{2}=\lim _{\theta \rightarrow 0}\left|J_{2}\left(0, \theta_{i}\right) / J_{2}(t, \theta)\right|^{1 / 2}=\left|\frac{\partial \theta_{i}}{\partial \theta}\right|^{1 / 2} .
$$

Therefore, at the boundary $r=r_{b}$, the three-dimensional amplitude is

$$
A=\left|\frac{\partial \theta_{i}}{\partial \theta_{f}}\right|
$$

(ii) The amplitude of the returning wave near the core is calculated by the standard method: The semiclassical returning wave is set equal to a constant $N$ times the closed-form expression (8.2):

$$
\psi_{\text {ret }}=N\left(\psi_{c, \theta_{f}=0}\right)_{\text {inc }} .
$$

We find

$$
\begin{aligned}
N & =\psi_{\text {out }}\left(r_{b}, 0\right) A \exp \left[i\left(S_{2}-\mu \pi / 2\right)\right] /\left[\frac{1}{2}\left[\frac{2}{\pi \sqrt{8 r}}\right]^{1 / 2} \exp \left[-i\left(\sqrt{8 r_{b}}-\pi / 4\right)\right]\right] \\
& =2^{2} \pi r_{b}^{-1 / 2} e^{-i \pi} e^{i 2 \sqrt{8 r_{b}}}\left|\frac{\partial \theta_{i}}{\partial \theta_{f}}\right| \exp \left[i\left(S_{2}-\mu \pi / 2-\pi / 2\right)\right] \mathscr{y}_{0}(0),
\end{aligned}
$$

where $\mathscr{y}_{0}(0)$ is given in Eqs. (5.13), (5.15), and (5.19) for $\mathrm{H}$, and "Pseudo-H," and $\mathrm{Na}$. The same constant $N$ multiplies the partial-wave expansion (7.3) of the returning wave close to the nucleus, and, as in Eq. (7.7),

$$
\left\langle D \psi_{i} \mid \psi_{\mathrm{Na}, \theta_{f}=0}\right\rangle=\frac{4 \pi}{\sqrt{2}} \tilde{\mathscr{F}}_{0}(0) .
$$

Finally, combining Eqs. (8.8), (8.10), and (8.11), straightforward algebra gives

$$
\begin{aligned}
& D f_{1}(E)= \sum_{n} \operatorname{Im}\left[C_{n} \exp \left(i \Delta_{n}\right)\right] \\
&=\operatorname{Im}\left|\sum_{n}\left(E-E_{i}\right) 2^{9 / 2} \pi r_{b}^{-1 / 2}\right| \frac{\partial \theta_{i}}{\partial \theta_{f}} \mid \\
& \quad \times \exp \left[i \left(S^{n}-\mu_{n} \pi / 2\right.\right. \\
&\left.-\pi / 2)] \widetilde{\mathscr{y}}_{0}(0) \mathscr{y}_{0}(0)\right],
\end{aligned}
$$

in which

$$
S^{n}=n\left(S_{2}+2 \sqrt{8 r_{b}}\right) .
$$

For $E>0$ in an electric field, the Maslov index on the $n$th return is $2 n-1$.

\section{CLASSICAL TRAJECTORIES AND SCALED VARIABLES}

Up to this point, we have dealt mainly with the quantum aspects of the problem: the initial state, and the out- going, returning, and scattered waves in the vicinity of the nucleus. The only statement we have made about the waves in the rest of space is that they can be described by a semiclassical approximation (6.1). In this section we describe classical trajectories in combined Coulomb and electric fields, and give formulas for the actions and amplitude $A(r)$ that are used in the semiclassical approximation. Most of the formulas are standard "textbook" results, and we only briefly summarize the essential ones.

\section{A. General properties}

The Hamiltonian of a hydrogenic atom in a homogeneous static electric field $F$ is

$$
H=\frac{1}{2}\left(p_{z}^{2}+p_{\rho}^{2}+l_{z}^{2} / \rho^{2}\right)-1 /\left(\rho^{2}+z^{2}\right)^{1 / 2}+F z .
$$

A transformation to scaled variables according to

$$
\begin{aligned}
& \hat{\rho}=\rho / \alpha, \quad \hat{z}=z / \alpha, \\
& \hat{p}_{\rho}=p_{\rho} / \beta, \quad \hat{p}_{z}=p_{z} / \beta, \quad \hat{t}=t / \gamma,
\end{aligned}
$$

with

$$
\alpha=F^{-1 / 2}, \quad \beta=F^{1 / 4}, \quad \gamma=F^{-3 / 4},
$$

then converts the Hamiltonian to the form

$\hat{H}=H / \beta^{2}=\hat{p}_{z}^{2} / 2+\hat{p}_{p}^{2} / 2+L^{2} / 2 \hat{\rho}^{2}-1 /\left(\hat{\rho}^{2}+\hat{z}^{2}\right)^{1 / 2}+\hat{z}$,

where the only parameter is $L$, the scaled component of 
angular momentum $L=l_{z} / \alpha \beta=m \hbar F^{-1 / 4}$. It follows that the shapes of all the trajectories depend only on the parameter $L$ and on the scaled energy $E / F^{1 / 2}$.

Defining parabolic coordinates and their corresponding canonical momenta,

$$
\begin{aligned}
& u=(\hat{r}+\hat{z})^{1 / 2}, \quad v=(\hat{r}-\hat{z})^{1 / 2}, \\
& \hat{z}=\frac{1}{2}\left(u^{2}-v^{2}\right), \quad \hat{\rho}=u v, \\
& p_{u}=\hat{p}_{\rho} v+\hat{p}_{z} u, \quad p_{v}=\hat{p}_{\rho} u-\hat{p}_{z} v, \\
& \hat{H}=\frac{1}{2} \frac{p_{u}^{2}+p_{v}^{2}-4}{u^{2}+v^{2}}+\frac{L^{2}}{2 u^{2} v^{2}}+\frac{1}{2}\left(u^{2}-v^{2}\right),
\end{aligned}
$$

we introduce an independent variable $\tau$ which connects to time $\hat{t}$ by [10]

$$
\frac{d \hat{t}}{d \tau}=u^{2}+v^{2}
$$

This change of independent variables from $t$ to $\tau(t)$ preserves the canonical form if the new effective Hamiltonian is defined as

$$
\begin{aligned}
\widetilde{H}= & \left(u^{2}+v^{2}\right)(\hat{H}-\varepsilon) \\
= & \frac{1}{2}\left(p_{u}^{2}+p_{v}^{2}\right)+\frac{1}{2}\left(u^{4}-v^{4}\right)+\frac{L^{2}}{2 u^{2}}+\frac{L^{2}}{2 v^{2}} \\
& -\varepsilon\left(u^{2}+v^{2}\right)-2 .
\end{aligned}
$$

As $u, v, p_{u}$, and $p_{v}$ evolve with the "fictitious time" $\tau, \widetilde{H}$ is conserved and its value is zero. [Note that $d u / d \tau=p_{u}$, but $d u / d \hat{t}=p_{u} /\left(u^{2}+v^{2}\right)$.] For the Hamiltonian (9.6b) the motion in the $u$ and $v$ directions can be separated:

$$
\begin{aligned}
& \frac{1}{2} p_{u}^{2}+\frac{1}{2} u^{4}-\varepsilon u^{2}+L^{2} / 2 u^{2}-1=\beta, \\
& \frac{1}{2} p_{v}^{2}-\frac{1}{2} v^{4}-\varepsilon v^{2}+L^{2} / 2 v^{2}-1=-\beta .
\end{aligned}
$$

The constant $\beta$ is a conserved quantity closely related to the Laplace (or Runge-Lenz) vector [11]

$$
\beta=-\left[L^{2} \hat{z} / \hat{\rho}^{2}+\hat{p}_{\rho}\left(\hat{z} \hat{p}_{\rho}-\hat{\rho} \hat{p}_{z}\right)-\hat{z} / \hat{r}\right]+\frac{1}{2} \hat{\rho}^{2} .
$$

In our case, trajectories all begin radially outward on a circle of radius $\widehat{r}_{i}$; hence, the term $\widehat{z} \hat{p}_{\rho}-\widehat{\rho} \widehat{p}_{z}$ initially vanishes. Then $\beta$ can be related to the initial position $\left(r_{i}, \theta_{i}\right)$ by the formula

$$
\beta=\cos \theta_{i}+\frac{1}{2} \widehat{r}_{i}^{2} \sin ^{2} \theta_{i}-L^{2} \cos \theta_{i} / \widehat{r}_{i} \sin ^{2} \theta_{i} .
$$

\section{B. Semiclassical phase and amplitude}

The change in phase of the semiclassical wave function on one cycle around the closed orbit is

$\hat{S}_{2}=\oint\left(p_{u} d u+p_{v} d v\right)$,

$\oint_{p_{u}} d u=\oint\left(2 \beta+2 \varepsilon u^{2}-u^{4}-L^{2} / 2 u^{2}+2\right)^{1 / 2} d u$,

$\oint_{p_{v}} d v=\oint\left(-2 \beta+2 \varepsilon v^{2}+v^{4}-L^{2} / 2 v^{2}+2\right)^{1 / 2} d v$.

The circle on the integral means that it is evaluated from the initial to the final surface. The "period" of the orbit (in fictitious time) will also be used below. It is

$$
\tau=\oint d u /\left(2 \beta+2 \varepsilon u^{2}-u^{4}-L^{2} / 2 u^{2}+2\right)^{1 / 2} .
$$

Likewise, the amplitude factor $A(\mathbf{r})$ of the semiclassical wave function can also be derived from Eqs. (9.7). The relevant Jacobian

$$
\begin{aligned}
J\left(t, \theta_{i}\right) & =\frac{\partial(x, y, z)}{\partial\left(t, \theta_{i}, \varphi_{i}\right)} \\
& =\left|\frac{\partial(x, y, z) \partial(\rho, z, \varphi) \partial(u, v, \varphi) \partial\left(\tau, \theta_{i}, \varphi_{i}\right)}{\partial(\rho, z, \varphi) \partial(u, v, \varphi) \partial\left(\tau, \theta_{i}, \varphi_{i}\right) \partial\left(t, \theta_{i}, \varphi_{i}\right)}\right| \\
& =u v J_{2}\left(\tau, \theta_{i}\right)
\end{aligned}
$$

where

$$
J_{2}\left(\tau, \theta_{i}\right)=\frac{\partial(u, v)}{\partial\left(\tau, \theta_{i}\right)} .
$$

The quantities $(\partial u / \partial \tau)_{\theta_{i}}$ and $(\partial v / \partial \tau)_{\theta_{i}}$ are, respectively, $p_{u}$ and $p_{v}$, which can be related to $u$ and $v$ through the conservation law (9.7). The other two derivatives $\left(\partial u / \partial \theta_{i}\right)_{\tau}$ and $\left(\partial v / \partial \theta_{i}\right)_{\tau}$ can be calculated as follows. On the initial surface,

$u_{i}=r_{i}^{1 / 2}\left(1+\cos \theta_{i}\right)^{1 / 2}, \quad v_{i}=r_{i}^{1 / 2}\left(1-\cos \theta_{i}\right)^{1 / 2}$,

so

$J_{2}\left(\tau=0, \theta_{i}\right)=\sqrt{r_{i} / 2}\left[\cos \left(\theta_{i} / 2\right)\left(2 \beta+2 \varepsilon u_{i}^{2}-u_{i}^{4}-L^{2} / 2 u_{i}^{2}+2\right)^{1 / 2}+\sin \left(\theta_{i} / 2\right)\left(-2 \beta+2 \varepsilon v_{i}^{2}+v_{i}^{4}-L^{2} / 2 v_{i}+2\right)^{1 / 2}\right]$.

At the final time, when the electron returns, these derivatives with respect to $\theta_{i}$ depend upon integrals over the whole trajectory. As the electron moves along its path, $\tau$ increases as

$\tau\left(u, \theta_{i}\right)=\int_{u i}^{u} d u /\left[2 \beta\left(\theta_{i}\right)+2 \varepsilon u^{2}-u^{4}-L^{2} / 2 u^{2}+2\right]^{1 / 2}$

So at the (fixed) final time of return to the boundary,

$$
\left(\frac{\partial u}{\partial \theta_{i}}\right]_{\tau}=-\left[\frac{\partial \tau}{\partial \theta_{i}}\right]_{u} /\left[\frac{\partial \tau}{\partial u}\right]_{\theta_{i}},
$$

and, similarly,

$$
\left[\frac{\partial v}{\partial \theta_{i}}\right]_{\tau}=-\left[\frac{\partial \tau}{\partial \theta_{i}}\right]_{v} /\left(\frac{\partial \tau}{\partial v}\right]_{\theta_{i}} .
$$

Numerical evaluation of these integrals gives $J_{2}\left(\tau_{f}, \theta_{i}\right)$, 
and the semiclassical amplitude is

$$
A=\left(u_{i} v_{i} / u_{f} v_{f}\right)^{1 / 2}\left[J_{2}\left(0, \theta_{i}\right) / J_{2}\left(\tau_{f}, \theta_{i}\right)\right]^{1 / 2} .
$$

\section{Evaluation for the special case $\theta=0$}

As stated before, when the angular momentum $L=0$, there is a closed orbit with $\theta=0$, and the motions of this closed orbit and its neighbor are mainly along the $u$ direction $(v \approx 0)$. The constant $\beta$ is

$$
\beta=\lim _{\theta_{i} \rightarrow 0}\left(\cos \theta_{i}+\frac{1}{2} \widehat{r}^{2} \sin ^{2} \theta_{i}\right) .
$$

The two-dimensional action (9.10a) has no $v$ integral,

$$
\widehat{S}(\varepsilon)=\oint\left(4+2 \varepsilon u^{2}-u^{4}\right)^{1 / 2} d u,
$$

and the fictitious period of the orbit is

$$
\tau(\varepsilon)=\oint d u /\left(4+2 \varepsilon u^{2}-u^{4}\right)^{1 / 2} .
$$

The neighbors of the closed orbit are slightly off the $u$ direction. Their motion in $v$ is sensitively dependent on $\theta$. The velocity in the $v$ direction is obtained from $(9.7 \mathrm{~b})$, and we can neglect the $O\left(v^{4}\right)$ term in that equation,

$$
\frac{d v}{d \tau}=\left[2 \varepsilon v^{2}+2\left(1-\cos \theta_{i}\right)\right]^{1 / 2} .
$$

Therefore,

$$
v\left(\tau, \theta_{i}\right)= \begin{cases}\sqrt{\left(1-\cos \theta_{i}\right) / \varepsilon} \sinh \left(\sqrt{2 \varepsilon} \tau+\operatorname{arcsinh} \sqrt{\widehat{r}_{i} \varepsilon}\right), & \varepsilon>0 \\ \sqrt{\left(1-\cos \theta_{i}\right) /|\varepsilon|} \sin \left(\sqrt{2|\varepsilon|}+\arcsin \sqrt{\hat{r}_{i}|\varepsilon|}\right), & \varepsilon<0\end{cases}
$$

At the boundary $u_{i}=u_{f}$ the amplitude at the final point on the orbit is

$$
\begin{aligned}
A & =\lim _{\theta_{i} \rightarrow 0}\left[\left[v_{i} \frac{\partial v_{i}}{\partial \theta_{i}}\right] /\left[v_{f} \frac{\partial v_{f}}{\partial \theta_{i}}\right]\right]^{1 / 2} \\
& = \begin{cases}\frac{\sqrt{\hat{r}_{b} / 2} \sqrt{2 \varepsilon}}{\sinh \sqrt{2 \varepsilon} \tau}, & \varepsilon>0 \\
\frac{\sqrt{\hat{r}_{b} / 2 \sqrt{2 \mid \varepsilon}}}{\sin \sqrt{2|\varepsilon| \tau}}, & \varepsilon<0\end{cases}
\end{aligned}
$$

When $\varepsilon=0$, then

$$
A=\frac{\sqrt{\hat{r}_{b} / 2}}{\tau} \text {. }
$$

Extending this argument, the amplitude on the $n$th return is obtained by replacing $\tau(\varepsilon)$ by $n \tau(\varepsilon)$. Similarly, the scaled action on the $n$th return is given by $n \hat{S}(\varepsilon)$. These are the quantities that belong in the formula (8.12):

$$
\begin{aligned}
D f_{1}(E)=\operatorname{Im}\left(\sum_{n}\right. & \left(E-E_{i}\right) 2^{9 / 2} \pi F^{1 / 4} \frac{\varepsilon^{1 / 2}}{\operatorname{Si}(\sqrt{2|\varepsilon|} n \tau)} \\
& \times \exp \left(i\left\{n\left[\widehat{S}(\varepsilon) F^{-1 / 4}-\pi\right]-v_{n} \pi / 2\right\}\right) \\
& \left.\times \widetilde{\mathscr{H}}_{0}(0) \mathscr{y}_{0}(0)\right]
\end{aligned}
$$

where $\operatorname{Si}(\sqrt{2|\varepsilon|} n \tau)$ means $\sin (\sqrt{2|\varepsilon|} n \tau)$ if $\varepsilon<0$ and $\sinh (\sqrt{2 \varepsilon} n \tau)$ if $\varepsilon>0$. Here the phase associated with the $n$th repetition of the closed orbit has three terms. The first term $n\left[\hat{S}(\varepsilon) F^{-1 / 4}\right]$ is simply the $n$th multiple of the classical action. The term $-n \pi$ comes from the following facts. Above $E=0$, there is a caustic at the turning point of the orbit and a kind of focus at the nucleus, so the Maslov index of the returning wave on the $n$th return is $2 n-1$. In addition, Eq. (8.12) contains another $\pi / 2$, so $-\mu_{n} \pi / 2-\pi / 2=n \pi$. The last term $\nu_{n} \pi / 2$ applies only for $E<0$. It counts the additional caustics that arise when the neighbors of the $\theta=0$ orbit cross over. The number of such crossings can be determined by examining Eq. (9.24):

$$
v_{n}=\left\{\begin{array}{l}
0, \quad \varepsilon>0 \\
2 \operatorname{Int}(n \alpha / \pi), \quad \varepsilon<0
\end{array}\right.
$$

where $\alpha=\sqrt{2|\varepsilon|} \tau(\varepsilon)$. This formula is finally reduced to

$$
\begin{aligned}
D f_{1}(E)=g(E) F^{1 / 4} \sum_{n} & \frac{\varepsilon^{1 / 2}}{\operatorname{Si}(\sqrt{2|\varepsilon|} n \tau)} \\
& \times \sin \left\{n\left[\hat{S}(\varepsilon) F^{-1 / 4}-\pi\right]\right. \\
& \left.\quad-v_{n} \pi / 2+\Delta\right\},
\end{aligned}
$$

where $g(E)$ and $\Delta$ are related to the radial dipole in-

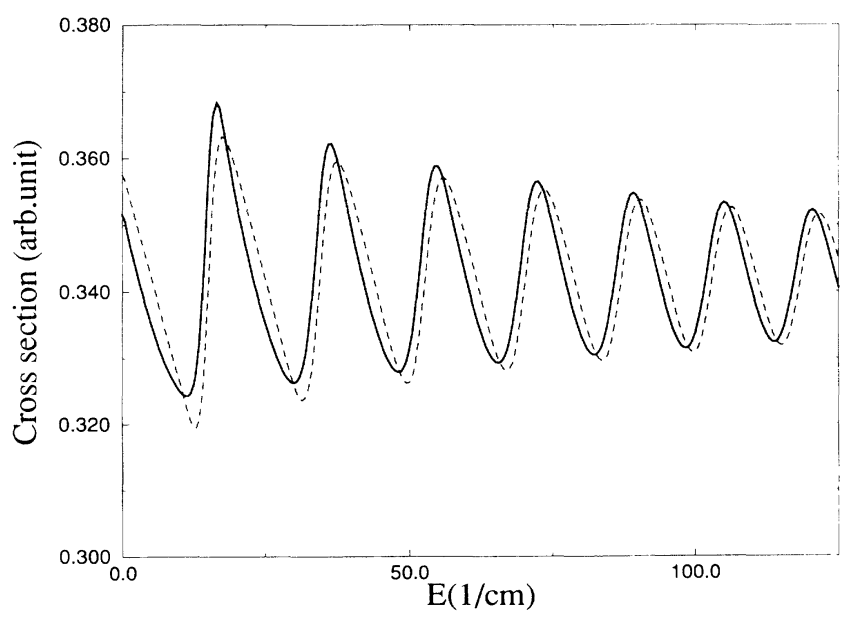

FIG. 3. Phase change of oscillations above threshold caused by phase shift associated with quantum defect. The solid line is the photoabsorption cross section for sodium; the dashed line is the cross section that would be obtained by setting $\Delta=0$. 
tegrals and quantum defects

$$
g(E)=\left(E-E_{i}\right) 2^{9 / 2} \pi\left|\widetilde{\mathscr{y}}_{0} \mathscr{Y}_{0}\right|, \quad \Delta=\arg \left(\widetilde{\mathscr{y}}_{0} \mathscr{Y}_{0}\right) .
$$

For $\mathrm{H}$ or Pseudo-H, $\widetilde{\mathscr{y}}_{0 \mathscr{F}_{0}}$ is a real number, so $\Delta=0$. For sodium, $\widetilde{\mathscr{y}}_{0} \mathscr{Y}_{0}$ is a complex number; by applying some simple complex algebra, we obtain the magnitude of $\tilde{\mathscr{H}}_{0} \mathscr{Y}_{0}$ as

$$
\left|\widetilde{\mathscr{y}}_{0} \mathscr{\mathscr { F }}_{0}\right|_{3 / 2}=2\left|\widetilde{\mathscr{y}}_{0} \mathscr{Y}_{0}\right|_{1 / 2}=1.618
$$

The phases of both states are the same,

$$
\Delta=\arg \left(\widetilde{y}_{0} \mathscr{y}_{0}\right)=0.5184 .
$$

Figure 3 displays the effect of the phase shift $\Delta$ on the absorption spectrum.

For $E>0$, Eq. (9.28) is compared with experiments in the accompanying paper.

\section{ACKNOWLEDGMENTS}

This research was supported by the National Science Foundation, the Office of Naval Research, and the Jeffress Foundation. We thank the Institute for Theoretical Atomic and Molecular Physics for their hospitality.
[1] J. Gao, J. B. Delos, and M. Baruch, preceding paper, Phys. Rev. A 46, 1449 (1992).

[2] M. L. Du and J. B. Delos, Phys. Rev. A 38, 1913 (1988).

[3] The closely related work of E. B. Bogomol'nyi, Zh. Eksp. Teor. Fiz. 96, 487 (1989), was already cited in Ref. [1]. We mention also the somewhat related work of V. D. Kondratovich and V. N. Ostrovsky, J. Phys. B 23, 21 (1990); 23, 3785 (1990).

[4] One may question whether closed-orbit theory is capable of describing the narrow resonances that are present for $E<0$. We have preliminary evidence that the theory can obtain locations of resonances. However, these questions are deferred to a future paper.

[5] David A Harmin, Phys. Rev. A 26, 2656 (1982).

[6] H. Rottke and K. H. Welge, Phys. Rev. A 33, 301 (1986); W. Sander, K. A. Safinya, and T. F. Gallagher, ibid. 23,
2448 (1981); T. S. Luk, Louis Dimauro, T. Bergeman, and Harold Metcalf, Phys. Rev. Lett. 47, 83 (1981).

[7] C. E. Moore, Atomic Energy Levels, Natl. Bur. Stand. (U.S.) Circ. No. 35 (U.S. GPO, Washington, D.C., 1971), Vol. 1.

[8] S. Levit, K. Möhring, U. Smilansky, and T. Dreyfus, Ann. Phys. (N.Y.) 114, 223 (1978).

[9] Scattering by a Coulomb field plus a core is treated by L. I. Schiff, Quantum Mechanics, 2nd ed. (McGraw-Hill, New York, 1955), Eq. (21.27).

[10] E. L. Stiefel and G. Scheifele, Linear and Regular Celestial Mechanics (Springer, New York, 1971).

[11] The constant $\beta$ turns out to be the same as that given in L. D. Landau, Mechanics.

[12] J. M. Mao and J. B. Delos, J. Stat. Phys. (to be published). 